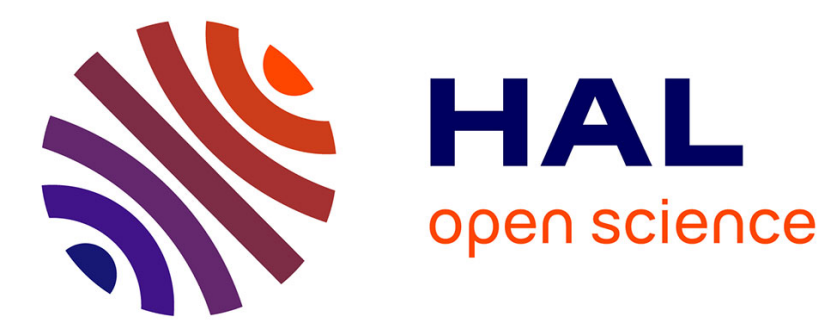

\title{
Randomized polynuclear growth with a columnar defect
} Vincent Beffara, Vladas Sidoravicius, Maria Eulalia Vares

\section{To cite this version:}

Vincent Beffara, Vladas Sidoravicius, Maria Eulalia Vares. Randomized polynuclear growth with a columnar defect. Probability Theory and Related Fields, 2010, 146 (3), pp.565-581. 10.1007/s00440009-0216-8 . ensl-00128386v2

\section{HAL Id: ensl-00128386}

https://hal-ens-lyon.archives-ouvertes.fr/ensl-00128386v2

Submitted on 3 Mar 2009

HAL is a multi-disciplinary open access archive for the deposit and dissemination of scientific research documents, whether they are published or not. The documents may come from teaching and research institutions in France or abroad, or from public or private research centers.
L'archive ouverte pluridisciplinaire HAL, est destinée au dépôt et à la diffusion de documents scientifiques de niveau recherche, publiés ou non, émanant des établissements d'enseignement et de recherche français ou étrangers, des laboratoires publics ou privés. 


\title{
Vincent Beffara · Vladas Sidoravicius . \\ Maria Eulalia Vares \\ Randomized polynuclear growth with a columnar defect
}

\begin{abstract}
We study a variant of poly-nuclear growth where the level boundaries perform continuous-time, discrete-space random walks, and study how its asymptotic behavior is affected by the presence of a columnar defect on the line. We prove that there is a non-trivial phase transition in the strength of the perturbation, above which the law of large numbers for the height function is modified.
\end{abstract}

Keywords Poly-nuclear growth · interacting random walks · zero-temperature Glauber dynamics · polymer pinning

Mathematics Subject Classification (2000) $60 \mathrm{~K} 35 \cdot 60 \mathrm{~K} 37$

\section{Preliminary considerations and statement of the results}

Rigorous study of growth processes, and constant attempts to give mathematical rigor to Kardar-Parisi-Zhang theory and its predictions, led to a very rich flow of results. Remarkable, but yet very limited progress was achieved in the last few years for $1+1$ dimensional models by using a broad spectrum of techniques and arguments from the theory of random matrices, first passage percolation and interacting particle systems. An important role in the successful application and interpretation of obtained results was played by the fact that some properties of

V. Beffara

UMPA - ENS Lyon, 46 Allée d'Italie, F-69364 Lyon Cedex 07, France

E-mail: vbeffara@ens-lyon.fr

V. Sidoravicius

CWI, Kruislaan 413, 1098SJ Amsterdam, The Netherlands and

IMPA, Estrada Dona Castorina 110, Rio de Janeiro RJ 22460-320, Brasil

E-mail: v.sidoravicius@cwi.nl, vladas@impa.br

M. E. Vares

CBPF, Rua Dr. Xavier Sigaud 150, Rio de Janeiro RJ 22290-180, Brasil

E-mail: eulalia@cbpf.br 
a particular $1+1$ dimensional growth could be mapped and studied in terms of the length of a maximal increasing sub-sequence in permutation of $N$ elements, with $N \rightarrow \infty$, or as properties of the one-dimensional totally asymmetric simple exclusion process, or finally as a directed polymer in two dimensions subject to a random potential.

The starting point and motivation of the present work was the following question: how can a localized defect, especially if it is small, affect the macroscopic behavior of growth system? This is one of the fundamental questions in non-equilibrium growth: is the asymptotic shape changed (faceted) in the macroscopic neighborhood of such a defect at any value of its strength, or, when the defect is too weak, then the fluctuations of the bulk evolution become predominant and destroy the effects of the obstruction in such a way that its presence becomes macroscopically undetectable?

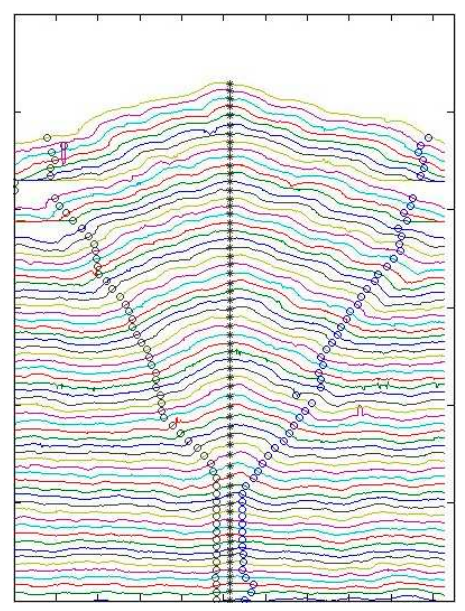

Fig. 1 Example: effect of the local defect on propagation of slow-combustion fronts: Higher concentration of potassium nitrate along vertical line changes shapes of otherwise nearly horizontal front lines. Higher velocity of propagation of combustion front along this line affects macroscopic picture producing a faceted shape around the defected region. (Experiment produced by M. Myllys et al. [12]. Reproduced by permission.)

Such a vanishing presence of the macroscopic effect as a function of the strength of obstruction represents what is called dynamic phase transition. The existence of such a transition, its scaling properties, the shape of the density profile near the obstruction, and also whether information percolates through the obstruction, are among the most important issues. As we already mentioned, in many cases these questions can be translated into the language of pinning of a directed polymer in presence of bulk disorder, or to the question of whether the presence of a static obstruction in a driven flow, such as a slow bond in one dimensional totally asymmetric simple exclusion process (TASEP), always results in change of the current, as predicted by mean-field theory (see [9]). 
Similar questions are often raised in the setting of first-passage percolation (FPP) (see [10] for a review). More specifically, consider an FPP process in which the passage times are shortened (in some random or deterministic way) along one of the axes. Such type of perturbation usually is referred in physics literature as columnar defect. Is it then true that the asymptotic shape of the cluster is always modified, or is there a phase transition for the amount of perturbation? Fig. 1 shows experimentally obtained successive slow-combustion fronts on the piece of paper, in the presence of the columnar defect (see [12] for details).

All these questions remain rather poorly understood on the experimental and the theoretical physics level, and essentially no rigorous mathematical results are available. "Tone particular model, however, known as Poly-Nuclear Growth (or PNG for short) makes the exception. In this process, on a flat substrate, layers appear on top of each other according to a Poisson process, and then grow laterally with constant speed -1 and +1 , respectively, and merging whenever they collide. This model is interesting in the sense that it lends itself to the explicit computation of various quantities, such as growth speed and interface fluctuations (see [4. 13]). The proofs depend on exact algebraic computations, and it is not clear how to apply them as soon as the dynamics are modified in a non-translation-invariant way (with an exception of certain symmetrized cases (see [5])). To treat the PNG case, new arguments have been developed in [7] and [G].

The process which we introduce and study in this paper, the Randomized PolyNuclear Growth (or RPNG for short), has several apparent similarities with PNG, but the substantial difference is that after the nucleation, boundaries of layers (plateaux) move as independent random walks instead of moving deterministically. This dynamics corresponds to the modified Glauber dynamics for the twodimensional Ising model on a solid surface at temperature 0 , as considered in [8], with additional nucleations. Informally it can be described as follows: On each edge of the discrete line $\mathbb{Z}$, with some positive rate $\lambda$, pedestals of width and height 1 appear. Every pedestal will evolve into a plateau, its endpoints performing independent continuous-time, discrete-space symmetric random walks with jump rate 1 , until one of the following happens:

- If two plateaux collide, i.e. if the right edge of one occupies the same site as the left edge of the next one, then they merge and only the two extremal endpoints continue to perform random walks;

- If one of the two endpoints of a plateau jumps to the location of the other one, so that the plateau shrinks to width 0 , then it vanishes.

If a pedestal appears on an edge which is already spanned by one of the plateaux, it appears on an additional layer on top of it. Only two plateaux evolving

\footnotetext{
${ }^{1}$ In this context it also interesting to mention a recent work (see [⿰丨丨), where alternative methods to detect localized inhomogeneity were used. In [2] the following question was studied: Consider a graph with a set of vertices and oriented edges connecting pairs of vertices. Each vertex is associated with a random variable and these are assumed to be independent. In this setting, the authors consider the following hypothesis testing problem: under the null, the random variables have common distribution $N(0,1)$, while under the alternative, there is an unknown path along which random variables have distribution $N(\mu, 1), \mu>0$, and distribution $N(0,1)$ away from it. The authors study for which values of the mean shift $\mu$ one can reliably detect the presence of such defected path and for which values this is impossible.
} 
within the same layer can merge, and besides the growth of the plateaux at different layers has to obey the following constraint: Every plateau which is not on the ground level must always lie entirely on top of a plateau at the layer below it. Each jump which would break this constraint is simply suppressed. For any fixed value of $\lambda$ (see Proposition 1 below) the process has linear growth, i.e. if by $h_{t}(e)$ we denote the height of the aggregate at time $t$ above the edge $e$, then the following limit exists:

$$
v(\lambda):=\lim _{t \rightarrow+\infty} h_{t}(e) / t>0 .
$$

Notice that in the case of a strictly growing process (such as first- or last-passage percolation), the law of large numbers is usually a direct consequence of a statement of sub-additivity, while if the dynamics is allowed to both grow and shrink, like in the present case, even the existence of the asymptotic speed is non-trivial.

Next we will introduce a "columnar defect" to the system by changing the nucleation rate on the edge $e_{0}:=<0,1>$ from $\lambda$ to $\lambda+\lambda_{0}$, with $\lambda_{0}>0$, and the main question which we address is how does this affect the asymptotic growth rate of the aggregate above the origin. More precisely, let $h_{t}^{\lambda_{0}}\left(e_{0}\right)$ be the height of the aggregate above $e_{0}$ at time $t$ under the modified dynamics (we make the dependency on $\lambda$ implicit in this notation, since the parameter of interest is $\lambda_{0}$ ). Assuming that the following limit exists

$$
v\left(\lambda, \lambda_{0}\right):=\lim _{t \rightarrow+\infty} h_{t}^{\lambda_{0}}\left(e_{0}\right) / t
$$

we are interested in whether the inequality

$$
v\left(\lambda, \lambda_{0}\right)>v(\lambda) \equiv v(\lambda, 0)
$$

holds for all $\lambda_{0}>0$ and set: $\lambda_{c}(\lambda):=\inf \left\{\lambda_{0}: v\left(\lambda, \lambda_{0}\right)>v(\lambda)\right\}$ be the critical value of $\lambda_{0}$.

We are now ready to state our main result.

\section{Theorem 1}

$$
v\left(\lambda, \lambda_{0}\right):=\lim _{t \rightarrow+\infty} \frac{h_{t}^{\lambda_{0}}\left(e_{0}\right)}{t}=\lambda+\max \left\{\lambda_{0}-1,0\right\}
$$

almost surely. In particular, $\lambda_{c}(\lambda)=1$, for any value of $\lambda$.

In proving this theorem, we actually obtain a more precise description of the behavior of the system in the supercritical phase $\lambda_{0}>\lambda_{c}(\lambda)$ : the behavior of $h_{t}^{\lambda_{0}}(e)$ for $e \neq e_{0}$ is perturbed only sub-linearly. In other words, the defect creates a kind of "antenna" at the origin. This contrasts with the expected behavior of the usual PNG with a supercritical localized defect, for which the region of influence of the additional nucleation process is believed to extend linearly in space. However if in the RPNG model we would remove assumption of the symmetry of walks and would take the boundaries of plateaux moving as random walks with a drift (left boundary with a negative drift and right boundary with positive one), we expect the behavior of the system to resemble the behavior of PNG.

Moreover, the phase transition is non-trivial even without nucleation in the bulk (i.e. $\lambda=0)$. Even in this simplified situation, for any value $\lambda_{0}>0$ the origin 
is eventually covered by plateaux for all times, however $0<\lambda_{c}^{\prime}:=\lambda_{c}(0)<+\infty$ so that if $\lambda_{0}<\lambda_{c}^{\prime}$ then $v\left(0, \lambda_{0}\right)=0$, i.e. there is sub-linear growth, while for $\lambda_{0}>\lambda_{c}^{\prime}$ we have $v\left(0, \lambda_{0}\right)>0$. As we shall see, $\lambda_{c}^{\prime}=1$, in agreement with Theorem 1 .

Comment It might be instructive to compare with the following simplified model in the half-line $\mathbb{R}_{+}$. Consider a situation corresponding to $\lambda=0$ and an additional vertical wall at vertex 0 to which the left endpoint of each pedestal is fixed: the initial configuration is empty; at each occurrence of a Poisson process in $\mathbb{R}_{+}$of intensity $\lambda_{0}$, a new pedestal is created at the edge $e_{0}$ - and this is the only source in the system. Right boundaries of pedestals move according to the same mechanism as described in the basic homogeneous model, except that now each pedestal is annihilated upon its right boundary hitting the site 0 .

Of particular interest is the particular case $\lambda_{0}=1 / 2$, when the system is in exact bijection with 0-temperature Glauber dynamics for the two-dimensional Ising model, with an initial configuration consisting in the first quadrant uniformly at spin -1 and the rest of the lattice uniformly at spin +1 . The height function at $e_{0}$, here represented by $N_{t}$, the number of pedestals in the system at time $t$, describes the shape of the $+/-$ interface, and in the large time limit it is known to behave according to Lifshits' law of motion by mean-curvature (see [8], [14]).

In this particular case, there is also a one-to-one correspondence with the simple symmetric exclusion process: $N_{t}$ corresponds to the position of the rightmost particle in a symmetric simple exclusion process in $\mathbb{Z}$ starting with the configuration where the occupied sites are exactly those $x \leq 0$. A classical result of Arratia (see Theorem 2 in [3], cf. also [11], comments at p. 414) tells in particular that $N_{t} / t \rightarrow 0$ almost surely. A simple comparison with this case tells us that $1 / 2$ is the critical value of $\lambda_{0}$ in this simplified model.

Structure of the paper. In Section 2 we give a formal construction of the process, in terms of a Harris-type representation, and discuss two other equivalent representations. In Section 3 we derive basic properties of the homogeneous process without the defect at $e_{0}$. More specifically, we prove a law of large numbers for the height of the interface above edge $e_{0}$; a key step towards this result is the proof of eventual covering of any finite-size interval of the line. Finally, in Section 4 we present the proof of Theorem 1.

\section{Construction of the process: three ways to look at the system}

\subsection{Description as an interacting particle system}

One can describe this process in terms of an interacting particle system. Each left endpoint of a plateau is represented by a left-particle or $\ell$-particle for short, at the corresponding site of $\mathbb{Z}$, and each right endpoint of a plateau as a right-particle, or $r$-particle (which can be interpreted as an anti-particle). If several plateaux have their endpoints at the same site, this site will contain several particles, but notice that a site will never contain particles of both types.

The dynamics then looks like a zero-range process with annihilation and nucleation, i.e.:

- For each non-empty site, with rate 1 , one of the particles jumps with equal probability to a neighboring site; and if the target site contains a particle of the 
other type, then the jumping particle and one of the target particles annihilate instantaneously;

- On every edge $(x, x+1)$ of $\mathbb{Z}$, with rate $\lambda$, there is nucleation, meaning that an $\ell$-particle appears at $x$ and an $r$-particle appears at $x+1$; in each case, the particle is instantaneously annihilated (together with a particle of the opposite type), if a particle of the opposite type is already present at the corresponding site.

Remark. Notice that the particle configuration at time $t$ gives only partial information about the initial system; in particular, it gives no way of following the evolution of a given plateau boundary as a function of time - indeed, with our description of the particle dynamics, the notion of a tagged particle is ill-defined. Nevertheless, knowing the particle configuration at time $t$ allows for the reconstruction of the plateaux configuration at the same instant up to a global height change; and of course, the full space-time history contains exactly the same information for both descriptions of the system.

We first construct the above described interacting particle system using Harris graphical representation. Let $\mathbb{E}:=\{\langle x, x+1\rangle, x \in \mathbb{Z}\}$ be the set of edges of the integer line $\mathbb{Z}$. Define the following three independent Poisson systems:

- $\mathscr{L}_{r}=\left\{\mathscr{L}_{r}(e), e \in \mathbb{E}\right\}$ is a collection of independent Poisson processes of leftarrows, each with intensity $\mathrm{d} t / 2$; the individual arrows (or marks) of the process at edge $e$ will be denoted by $\mathscr{L}_{r}^{n}(e), n \geqslant 1$, with similar notations for the other two systems;

- $\mathscr{L}_{\ell}=\left\{\mathscr{L}_{\ell}(e), e \in \mathbb{E}\right\}$ is a collection of independent Poisson processes of leftarrows, each with intensity $\mathrm{d} t / 2$;

- $\mathscr{R}_{r}=\left\{\mathscr{R}_{r}(e), e \in \mathbb{E}\right\}$ is a collection of independent Poisson processes of rightarrows, each with intensity $\mathrm{d} t / 2$;

- $\mathscr{R}_{\ell}=\left\{\mathscr{R}_{\ell}(e), e \in \mathbb{E}\right\}$ is a collection of independent Poisson processes of rightarrows, each with intensity $\mathrm{d} t / 2$;

- $\mathscr{N}=\left\{\mathscr{N}_{e}, e \in \mathbb{E}\right\}$ is a collection of independent Poisson processes of undirected edges, each with intensity $\lambda \mathrm{d} t$

$\mathscr{L}_{r}$ (respectively $\mathscr{L}_{\ell}$ ) will correspond to jumps of right particles (respectively left particles) to the left, $\mathscr{R}_{r}$ and $\mathscr{R}_{\ell}$ to jumps of right and, respectively, left particles to the right, and $\mathscr{N}$ to nucleation times.

Given $T>0$, we first construct the process up to time $T$, as follows. There is positive density of edges carrying no mark of any of the Poisson before time $T$; between any two such edges, there are almost surely only finitely many marks appearing by time $T$, so that they can be ordered chronologically, and we only need to describe the action of each of the marks.

- If there is a directed arrow of type $r$ or, respectively, type $\ell$ at edge $e$ and time $t$, and if at this time the site at the source of the arrow is not empty and contains at least one right or, respectively, at least one left particle, then one particle at this site jumps in the direction of the arrow; moreover, if after this jump it meets a particle of the opposite type, they annihilate instantaneously.

- If there is a mark of $\mathscr{N}$ at edge $e=<x, x+1>$ and time $t$, then at this time an $\ell$-particle appears at site $x$ and an $r$-particle appears at site $x+1$; in each case if the corresponding site contain particles of the opposite type, a pair of opposite type particles is annihilated instantaneously. 
It is then easy to see that the obtained processes for different values of $T$ are consistent, and letting $T$ go to infinity gives the process for all time. We will refer to this version of the construction as the process in quenched environment, i.e. seeing the Poisson processes as a random scenery in which a deterministic process evolves.

Remark. Just for the sake of constructing of the process, there is no need to distinguish between $\mathscr{L}_{r}$ and $\mathscr{L}_{\ell}$, or $\mathscr{R}_{r}$ and $\mathscr{R}_{\ell}$. The distinction plays a role only when describing the evolution of the symmetric difference between two configurations, which we shall need later.

Particle system corresponding to the process with a columnar defect: A columnar defect in the plateaux dynamics, discussed in the Introduction, is described in the interacting particle system formalism by the addition an auxiliary nucleation process $\mathscr{N}_{0}$ with rate $\lambda_{0}>0$ localized on the edge $e_{0}$. In other words, the pedestals appear with rate $\lambda$ everywhere except at $e_{0}$ where the nucleation rate is $\lambda+\lambda_{0}$.

The construction of the new process can be done in exactly the same way as for the unperturbed system, using a graphical construction and letting the marks of $\mathscr{N}_{0}$ act the same way as those of $\mathscr{N}$.

Notation: $n_{x}(t)$ will denote the number of particles at site $x$ and time $t$ in the above construction; $\mathscr{N}_{e}(t)$ denotes the number of nucleation marks occurring at the edge $e$ up to time $t$.

\subsection{Construction in terms of interacting random walks}

In this subsection we will give an alternative interacting-random-walks-type description of our system. To do so, one needs a rule to determine how do these walks interact, and which of the particles at a given site will jump when an arrow (in the previous construction) appears, and which will be annihilated when a particle of the opposite type arises. Obviously the process itself will not be affected by the choice, so we have some freedom here. Some of the more natural choices are to declare that the oldest particle (i.e. the one that appeared earliest in the system), or the youngest particle, or the one that has been at the given site for the longest time, or the shortest time, is "active". The rule can even be different for jumps and annihilations. We will refer to such a rule as a priority rule.

In particular, the previous graphical construction can be replaced with the following interpretation of the dynamics:

1) Evolution of a single particle. Whenever a particle $i$ is born into the system, it comes together with a "project", i.e. a family $\left(T_{i}^{n}\right)$ of independent exponential times of rate 1 and a family $\left(\varepsilon_{i}^{n}\right)$ of independent signs, and a clock. Those will represent waiting times between consecutive jumps, and, respectively, directions of jumps for the path of this particle, if it were moving alone in an otherwise empty space.

2) Interaction. Choose any priority rule. When a particle is alone, it is always "active", i.e. its clock runs deterministically with speed 1 , and it executes its project, i.e. jumps according to times and directions prescribed in its project. When there are several particles at a given site, only one of them is active, according to the chosen rule, and the others are "sleeping". The clock of the active particle runs deterministically with speed 1 , the clocks of the other particles do not run at all. 
Whenever the clock of any particle reaches the next waiting time for the project of this particle, the particle jumps and the clock is reset.

Remark. Another natural choice for a jumping rule could be that at a site with $n$ particles, the clock of each of them runs with speed $1 / n$; again the distribution of the numbers of particles at different sites would be the same as in the graphical construction.

The formal construction of this dynamics could be done similarly as in the Harris construction: Given a space-time configuration of particles (space time Poisson process, corresponding to the $\mathscr{N}$ system in the graphical construction) and their families of "projects", for any fixed $T>0$, there are infinitely many edges to the left and to the right of the origin such that by time $T$ they will not be crossed by any type of particle, neither a nucleation will occur at them by time $T$. It follows quite simply that the evolution of the system within the space time block in between two such edges and $[0, T]$ depends only on finitely many particles, being independent from the evolution outside.

\subsection{Back to the PNG-like description}

We saw how to go from a PNG-like description to a family of interacting particles, but our final goal is to describe the long term behavior of the height (i.e. the number of layers) at a given edge, so we need a way to go back to the layer structure. As we saw already the $\ell-$ particles correspond to left boundaries of plateaux, and the $r$-particles correspond to right boundaries. It only remains to compute the height at edge $e_{0}=\langle 0,1\rangle$.

Let $\left(\phi_{t}^{\ell}\right)$ be the flow of $\ell$-particles through the edge $e_{0}: \phi_{0}^{\ell}=0$ and $\phi^{\ell}$ increases (resp. decreases) by 1 whenever an $\ell$-particle crosses $e_{0}$ to the right (resp. to the left). Define $\left(\phi_{t}^{r}\right)$ similarly as the flow of $r$-particles. Let $h_{t}\left(e_{0}\right)$ be the height of the profile at edge $e_{0}$ and time $t+$ (i.e., the càdlàg version in time of the height process). Then, recalling previous notation,

$$
h_{t}\left(e_{0}\right)=\mathscr{N}_{e_{0}}(t)-\phi_{t}^{\ell}+\phi_{t}^{r},
$$

From $h_{t}\left(e_{0}\right)$ and the previous discussion, it is then easy to obtain the whole profile of plateaux.

Remark. The graphical method gives us a construction of $h_{t}:=\left\{h_{t}(e)\right\}_{e \in \mathbb{E}}$ as a Markov process. The $\left\{h_{t}\right\}$-evolution is attractive (or monotone in the sense of definition 2.3 of Chap. II in [11]), though the particle system is not. The height process is also monotone in the nucleation rates.

3 Behavior of the homogeneous process $\left(\lambda>0, \lambda_{0}=0\right)$

3.1 Level lines, Hammersley-process-like description

Let $L_{t}$ (resp. $R_{t}$ ) be the position of the left (resp. right) end of the first-layer plateau containing $e_{0}$ at time $t$, if there is one i.e. if $h_{t}\left(e_{0}\right) \geqslant 1-$ for completeness, one can let $L_{t}=+\infty$ and $R_{t}=-\infty$ if $h_{t}\left(e_{0}\right)=0$. 
Let $\tau$ be the first time at which $R_{t}=L_{t}+2$, if any (otherwise $\tau=+\infty$ ). In the absence of other layers and of further nucleation, the behavior after $\tau$ of $L_{t}$ and $R_{t}$ until the first time when $R_{t}=L_{t}+1$ would exactly be that of two independent, centered continuous-time random walks. The presence of the second and above layers, by the previous description, can only affect the pair by the suppression of left jumps of $R_{t}$ and right jumps of $L_{t}$ - notice that if, in the description at Subsection 2.1, there are several particles at $R_{t}\left(L_{t}\right)$ at time $t$ and one has to jump to the right (left, resp.), then so does $R_{t}$ ( $L_{t}$ resp.).

Moreover, with rate $\lambda$ there is nucleation on the edge $\left\langle R_{t}, R_{t}+1\right\rangle$, and when this occurs, $R_{t}$ jumps to the right by at least one step. The last thing that can affect $R_{t}$ is if the plateau $\left[L_{t}, R_{t}\right]$ merges to the next plateau to its right, which again can only increase the value of $R_{t}$. The same remarks apply mutatis mutandis to $L_{t}$.

Taking all those effects into account, one obtains that the process $\left(R_{t}\right)$ stochastically dominates the biased random walk $\left(\tilde{R}_{t}\right)$ that has rates $1 / 2$ to jump to the left and $1 / 2+\lambda$ to jump to the right. Similarly, $\left(L_{t}\right)$ is stochastically dominated by $\left(\tilde{L}_{t}\right)$ which is biased to the left. Since the probability

$$
p=P\left(\forall t>0, \tilde{R}_{t}-\tilde{L}_{t}>1\right)
$$

is positive, using standard arguments we see that for $t_{0}$ large enough we have

$$
P\left(\forall t \geqslant t_{0}, R_{t}>\frac{\lambda t}{2} \text { and } L_{t}<-\frac{\lambda t}{2}\right)>1-c_{1} e^{-c_{2} t_{0}},
$$

for suitable positive constants $c_{1}, c_{2}$. Applying the Borel-Cantelli Lemma, one in particular sees that a.s. any compact interval of the real line will eventually remain covered by the plateau $\left[L_{t}, R_{t}\right]$. Moreover, extending the same line of reasoning one sees that

$$
\liminf _{t \rightarrow \infty} \frac{1}{t} \log R_{t} \geq C \quad \text { a.s. }
$$

for suitable constant $C>0$.

We shall give an additional description of the time evolution of the system, in terms of a family of space-time curves similar to a Hammersley process (cf. [1]]). The main difference is that the paths of the particles between meeting points are random walks instead of straight lines.

More precisely, one can see the path of any given particle, from its birth to its eventual annihilation, as a sequence of vertical and horizontal line segments. Let $\mathscr{C}_{1}$ be the union of the space-time paths of all the particles corresponding to endpoints of plateaux of the first layer, together with the segments joining the birth places in space-time of the corresponding pairs.

As we have seen above, for each bounded segment $[-K, K]$ there is an a.s. finite random time $t_{K}$, such that, the segment $[-K, K]$ will be covered by a plateau of the first level for all $t \geq t_{K}$. This means that $\mathscr{C}_{1}$ is the disjoint union of a countable family of finite loops (corresponding to plateaux with a finite life span) and one unbounded curve, which we will denote by $C_{1}$ and call the first level line of the process.

Similarly, let $\mathscr{C}_{k}$ be the union of all the space-time paths of the endpoints of plateaux of the $k$ th layer together with the segments joining the birth places in 


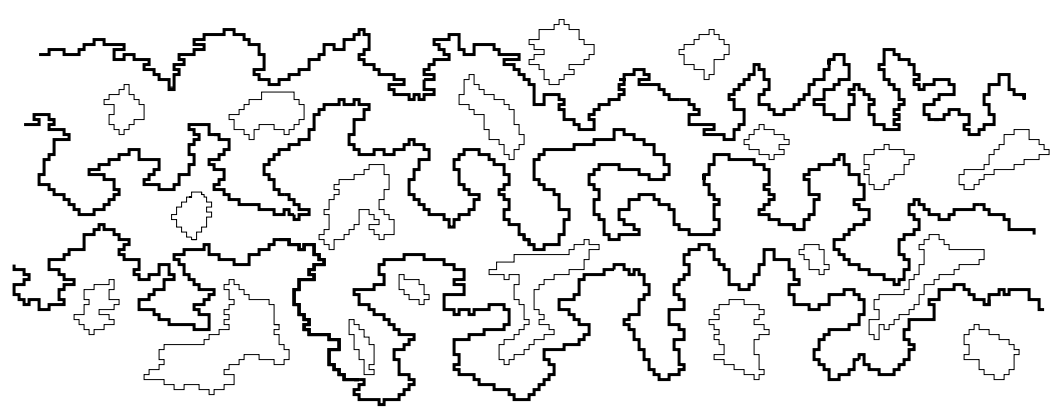

Fig. 2 Illustration of an RPNG process. (Level lines are bold.)

space-time of the corresponding pairs. $\mathscr{C}_{k}$ is then the disjoint union of a countable family of finite loops, and one unbounded curve $C_{k}$. Notice that in general a component of $\mathscr{C}_{k}$ and a component of $\mathscr{C}_{l}$ for $l \neq k$ need not be disjoint.

Using this description, it is possible to compute the height of the RPNG process at any edge $e$ and (almost) any time $t$ : Indeed, it is equal to the number of level lines that separate $(e, t)$ from the line $t=0$ (i.e. that pass below it an odd number of times), plus the number of loops containing $(e, t)$. This means that the law a of large numbers for $h_{t}$ can be translated into a statement about the asymptotic density of the level lines.

\subsection{The asymptotic speed}

The first step in proving a strong law of large numbers for the homogeneous process is to obtain an a priori bound for the number of particles present at a given site, at a given time. We obtain such a bound in the form of a stochastic domination result.

Lemma 1 There exist three countable families $\left(X_{\ell,}^{x}\right)_{x \in \mathbb{Z},}\left(X_{r,}^{x}\right)_{x \in \mathbb{Z}}$ and $\left(Y^{x}\right)_{x \in \mathbb{Z}}$ of integer-valued stochastic processes, and a coupling of them with the homogeneous particle system introduced above satisfying the following conditions:

1. For each $x \in \mathbb{Z},\left(X_{\ell, t}^{x}\right)_{t \geqslant 0}$ and $\left(X_{r, t}^{x}\right)_{t \geqslant 0}$ are simple symmetric random walks on $\mathbb{Z}_{+}$, with reflection at the origin, jump rate 1 at the origin and 2 elswhere, and $\left(Y_{t}^{x}\right)_{t \geqslant 0}$ is a simple symmetric random walk on $\mathbb{Z}$ with jump rate $\lambda$;

2. Almost surely, for all $x \in \mathbb{Z}$ and $t \geqslant 0$,

$$
n_{x}(t) \leqslant X_{\ell, t}^{x}+X_{r, t}^{x}+\sup _{s, s^{\prime} \in[0, t]}\left(Y_{s}^{x}-Y_{s^{\prime}}^{x}\right) .
$$

Proof Let $x \in \mathbb{Z}$, and in the graphical construction of the process (cf. Section 2), let $\left(T_{\ell, x, k}\right)_{k>0}$ (resp. $\left.\left(T_{\ell, x, k}^{\prime}\right)\right)$ be the $k$ th time at which there is an $\ell$-arrow pointing toward (resp. away from) site $x$. Define $X_{\ell}^{x}$ as follows: $X_{\ell, 0}^{x}=0, X_{\ell}^{x}$ jumps up by 1 at each $T_{\ell, x, k}$, and jumps down by 1 at each $t \in\left\{T_{\ell, x, k}^{\prime}\right\}$ such that $X_{\ell, t-}^{x}>0$. Clearly, 
$X_{\ell}^{x}$ defines a continuous-time random walk as in the statement. Similarly we define $X_{r}^{x}$ using $r$-arrows. Let $Y_{t}^{x}=\mathscr{N}_{\langle x, x+1\rangle}(t)-\mathscr{N}_{\langle x-1, x\rangle}(t)$.

$n_{x}$ can change for two reasons (nucleation or jumps): when a particle at $x$ jumps away, $n_{x}$ decreases by 1 , and when a particle at $x \pm 1$ jumps onto $x$, it either increases by 1 , or (if $n_{x}>0$ ) it decreases by 1 depending on the types of particles involved; if there is nucleation at the edge $\langle x-1, x\rangle$ or $\langle x, x+1\rangle$, then $n_{x}$ again increases or decreases by one according to the types of particles involved, as before.

Fix $t>0$, and let

$$
\tau:=\sup \left\{s<t: n_{x}(s)=0\right\} .
$$

If $\tau=t$ and $n_{x}(t)=0$ we have nothing to prove. From now on we are assuming that this is not the case. In particular, during the time interval $[\tau, t]$, the site $x$ is never empty and the type of particles present in it does not change; besides, $n_{x}(\tau-)=0$.

Apply the particle-counting of the previous paragraph between times $\tau$ and $t$. The contribution from nucleations is exactly equal to $\pm\left(Y_{t}^{x}-Y_{\tau-}^{x}\right)$ (where the sign depends on the type of particles present at site $x$ ), and the contribution from jumps is controlled by the jumps of $X_{\ell, t}^{x}$ or $X_{r, t}^{x}$. This leads to the following estimate:

$$
n_{x}(t) \leqslant\left|Y_{t}^{x}-Y_{\tau-}^{x}\right|+X_{\ell, t}^{x}+X_{r, t}^{x} .
$$

The bound in the statement of the Lemma directly follows.

Proposition 1 Recall that $e_{0}=<0,1>$. For every $\lambda>0$, almost surely,

$$
\lim _{t \rightarrow \infty} \frac{h_{t}\left(e_{0}\right)}{t}=\lambda .
$$

Proof Let $Q>0$ be a positive integer, and let

$$
H_{t}(Q):=\sum_{x=-Q}^{Q-1} h_{t}(<x, x+1>)
$$

be the integrated height of the process over the interval $[-Q, Q]$. The variations of $H_{t}(Q)$ in time come from several sources (according to the definition of the height process):

- Whenever there is a nucleation on one of the edges in $[-Q, Q], H_{t}(Q)$ increases by 1 ;

- Whenever an $\ell$-particle in $[-Q+1, Q-1]$ jumps to the right (resp. to the left), $H_{t}(Q)$ decreases (resp. increases) by 1

- Whenever an $r$-particle in $[-Q+1, Q-1]$ jumps to the left (resp. to the right), $H_{t}(Q)$ decreases (resp. increases) by 1 ;

- Whenever a particle sitting at $Q$ (resp. $-Q$ ) jumps to site $Q-1$ (resp. $-Q+1$ ), $H_{t}(Q)$ changes by +1 or -1 according to the type of particle $(\ell$ or $r$ ) involved.

Any other jump or nucleation might change the value of $n_{ \pm Q}(t)$, but it will never affect the value of $H_{t}$. Notice in the second and third cases, the decrease of $H_{t}(Q)$ occurs independently of the jump leading to an annihilation or not.

Notice that a particle sitting in $[-Q+1, Q-1]$ jumps to the left and to the right with the same rate (be it an $\ell$ - or an $r$-particle), and that the total jump rate 
of particles in this interval is, by construction, bounded by $2 Q-1$ (the total jump rate of particles at any given site being 1 if the site is non-empty, and of course 0 if the site is empty). Hence, the contribution of such jumps to $H_{t}(Q)$ is at most of the order of the position at time $t$ of a symmetric, continuous-time random walk jumping with rate $2 Q-1-i . e$. , they are of order $\sqrt{t}$.

What remains is the contribution of nucleations inside $[-Q, Q]$, which have total rate $2 Q \lambda$ and satisfy a strong law of large numbers, and the fourth term which, although difficult to estimate precisely due to its dependency on the exact particle distribution, only contributes to $H_{t}(Q)$ with rate at most 1 (i.e. , the sum of the jump rates from $-Q$ to $-Q+1$ and from $Q$ to $Q-1)$.

Summing all the above contributions to $H_{t}(Q)$, and then letting $t$ go to $+\infty$, we obtain with probability 1 :

$$
2 Q \lambda-1 \leqslant \liminf _{t \rightarrow \infty} \frac{H_{t}(Q)}{t} \leqslant \limsup _{t \rightarrow \infty} \frac{H_{t}(Q)}{t} \leqslant 2 Q \lambda+1 .
$$

Now, we know that at time $t$, the difference in height between two neighboring edges, say $\langle x-1, x\rangle$ and $\langle x, x+1\rangle$, only depends on the number (and type) of particles present at site $x$ at time $t$. By (7) we see that almost surely, for every $x \in \mathbb{Z}$,

$$
\lim _{t \rightarrow \infty} \frac{n_{x}(t)}{t}=0
$$

The height $h_{t}(e)$ at any edge $e \in[-Q, Q]$ can be computed from the height at edge $e_{0}$ by only looking at the values of the $n_{x}(t)$ for $x \in[-Q+1, Q-1]$. In particular, for such an $e$,

$$
\left|h_{t}(e)-h_{t}\left(e_{0}\right)\right| \leqslant \sum_{x=-Q+1}^{Q-1}\left|n_{x}(t)\right| .
$$

Summing over $e$ to obtain $H_{t}(Q)$, and taking a crude upper bound, leads to:

$$
\left|H_{t}(Q)-2 Q h_{t}\left(e_{0}\right)\right| \leqslant 2 Q \sum_{x=-Q+1}^{Q-1}\left|n_{x}(t)\right|
$$

Combining this with equation (8) and the previous estimate on the $n_{x}(t)$, we obtain, still with probability 1 :

$$
2 Q \lambda-1 \leqslant \liminf _{t \rightarrow \infty} \frac{2 Q h_{t}\left(e_{0}\right)}{t} \leqslant \limsup _{t \rightarrow \infty} \frac{2 Q h_{t}\left(e_{0}\right)}{t} \leqslant 2 Q \lambda+1
$$

(notice that we are still working with fixed $Q$ ). Dividing by $2 Q$, we get

$$
\lambda-\frac{1}{2 Q} \leqslant \liminf _{t \rightarrow \infty} \frac{h_{t}\left(e_{0}\right)}{t} \leqslant \limsup _{t \rightarrow \infty} \frac{h_{t}\left(e_{0}\right)}{t} \leqslant \lambda+\frac{1}{2 Q} .
$$

Since this holds for every positive $Q$, the proposition is proven. 


\section{Proof of Theorem 1}

We start with a homogeneous RPNG process as described in Section 2; let $h_{t}$ be its height function. Notice that the whole dynamics can be described in terms of this function. Notation: $e_{x}=\langle x, x+1\rangle$, for each integer $x$.

Now add an extra nucleation with rate $\lambda_{0}$ at the edge $e_{0}$ to the Harris graph of Section 2, and let $h_{t}^{\prime}$ be the height function of the modified process, with $n_{x}^{\prime}$ being the number of particles at $x$ in the corresponding new particle system. By monotonicity of the plateaux dynamics (cf. Remark right after equation (4)), we have that $h_{t}^{\prime} \geqslant h_{t}$; and let $\tilde{h}_{t}=h_{t}^{\prime}-h_{t} \geqslant 0$ be the discrepancy process. We are interested in differences in the law of large numbers between the two versions of the process, i.e. in the asymptotic properties of $\tilde{h}_{t} / t$.

The discrepancy process can be thought as a particle system, composed of what we call virtual particles: According to the difference

$$
\tilde{h}_{t}\left(e_{x-1}\right)-\tilde{h}_{t}\left(e_{x}\right)
$$

being positive or negative we say that at time $t$ there are

$$
\tilde{n}_{x}(t):=\left|\tilde{h}_{t}\left(e_{x-1}\right)-\tilde{h}_{t}\left(e_{x}\right)\right|
$$

virtual right or, respectively, virtual left particles at site $x$. In this interpretation the creation of virtual particles corresponds to a nucleation in $\mathscr{N}_{e_{0}}^{\prime} \backslash \mathscr{N}_{e_{0}}$.

Using the Harris description of subsection 2.1 (or the random walk representation of subsection 2.2) one can check explicitly that the spatial displacements of virtual particles remain as of independent random walks even in presence of the interaction with other virtual particles while jump times can be possibly delayed - or in other words, that conditionally on the whole particle system, the current rates of jump of a given virtual particle to the left or to the right always remain equal. Doing so in full detail involves looking at many different cases, but the discussion can be shortened considerably by the following considerations:

- If a site contains only virtual particles, then it is obvious from the construction that the first jump of one of them away from the site occurs with equal rate to the left and to the right; so we only need to check what happens when normal and virtual particles share a site.

- If a site contains both normal and virtual $\ell$-particles, then the perturbed system also contains $\ell$-particles. In particular, the original and perturbed system will use the same arrows in the Harris construction, meaning that their difference will not be affected by the dynamics and the virtual particles will not move as long as there are normal particles present, at which point we are back to the previous case. Of course, this also applies to the case of $r$-particles.

- If a site $x$ contains $a>0$ normal $\ell$-particles and $b>0$ virtual $r$-particles, then one needs to check 3 sub-cases $(a<b, a=b$ and $a>b)$, leading to different behavior in the perturbed system. We treat one of them fully, the others being similar: Assume that $b>a$ (see Fig. 3 ), so that the perturbed system contains $(b-a) r$-particles at site $x$. The occurrence of an arrow from $x$ affecting leftparticles will affect the unperturbed system and not the perturbed one, meaning that one of the virtual particles will follow the jump as well; the occurrence of 
an arrow affecting right-particles will only modify the perturbed system, and again in this case a virtual particle will follow; in both cases, the symmetry of the arrow processes translates into symmetry for the jumps of virtual particles.

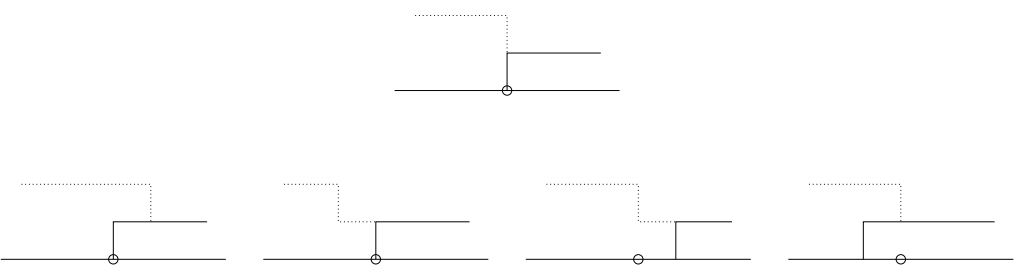

Fig. 3 Comparison of the perturbed and unperturbed dynamics in the case $b>a$. In plain lines are the height functions for the unperturbed system, and in dotted lines are the height functions for the perturbed one. On top is the initial configuration, and below are configurations after occurrence of an arrow in $\mathscr{R}_{r}, \mathscr{L}_{r}, \mathscr{R}_{\ell}$, and $\mathscr{L}_{\ell}$, respectively. The circle on the axis indicates the location of site $x$.

Proof of the Theorem. Due to the monotonicity, the height of the process with additional nucleation at the origin stochastically dominates that of the homogeneous process. In particular, we already know that almost surely

$$
\liminf \frac{h_{t}^{\prime}\left(e_{0}\right)}{t} \geqslant \lambda
$$

Step 1. Define

$$
\Delta_{t}=h_{t}^{\prime}\left(e_{0}\right)-\max \left\{h_{t}^{\prime}\left(e_{-1}\right), h_{t}^{\prime}\left(e_{1}\right)\right\} .
$$

If $\lambda_{0}>3+\lambda$, then

$$
\liminf _{t \rightarrow \infty} \frac{\Delta_{t}}{t}>0
$$

Indeed, if $\Delta_{t}>0$, the following can happen:

- if $h_{t}^{\prime}\left(e_{-1}\right)=h_{t}^{\prime}\left(e_{1}\right)$, then $\Delta_{t}$ may increase by one only because of a nucleation at the edge $e_{0}$, which happens at rate $\lambda+\lambda_{0}$, and it decreases by one either when some particle jumps away from vertex 0 or 1 , which happens with rate 2 , or when nucleation occurs at one of edges $e_{-1}$ or $e_{1}$, which happens with rate $2 \lambda$, or if a particle jumps from vertex -1 (resp. from vertex 2 ) to 0 (resp. to 1 ), which happens at rate 1 (if such a particle is present);

- if $h_{t}^{\prime}\left(e_{-1}\right)<h_{t}^{\prime}\left(e_{1}\right)$, then $\Delta_{t}$ may increase by one either because of a nucleation at the edge $e_{0}$, which happens at rate $\lambda+\lambda_{0}$, or because of a jump of a particle from vertex 2 to vertex 1 , provided a particle exists at vertex 2 , and which happens at rate $1 / 2$. On the other hand $\Delta_{t}$ decreases by one either if a particle jumps away from vertex 1 , which happens at rate 1 , or if a particle jumps from vertex 0 to 1 , which happens at rate $1 / 2$, or, finally, if nucleation occurs at the edge $e_{1}$, which happens at rate $\lambda$. 
Examining similarly the cases when $\Delta_{t}=0$ and $\Delta_{t}<0$, we obtain that $\Delta_{t}$ increases by one with rate at least $\lambda+\lambda_{0}$ and decreases by one with rate at most $3+2 \lambda$, and (9) easily follows.

Remark. In what follows we want to consider the unperturbed system as a random environment on which the virtual particles will evolve.

Step 2. From Proposition 1 and (9) it follows that if $\lambda_{0}>3+\lambda$, then

$$
\liminf _{t \rightarrow \infty} \frac{1}{t}\left(\tilde{h}_{t}\left(e_{0}\right)-\max \left\{\tilde{h}_{t}\left(e_{-1}\right) \tilde{h}_{t}\left(e_{1}\right)\right\}\right)>0
$$

and, in particular there exists $T_{*}<+\infty$ a.s. such that the number $\tilde{n}_{0}(s)$ of left virtual particles at the vertex 0 and the number $\tilde{n}_{1}(s)$ of right virtual particles at vertex 1 is positive, for all $s>T_{*}$. This also means that no virtual left (resp. right) particle created after $T_{*}$ enters $[2,+\infty)$ (resp. $\left.(-\infty,-1]\right)$.

Step 3. If $\lambda_{0}>3+\lambda$, then the total number $\sum_{k=2}^{+\infty} \tilde{n}_{k}(t)$ of virtual (in particular virtual right) particles in $[2,+\infty)$ grows sub-linearly in $t$. Indeed, if $\lambda_{0}>3+\lambda$, then from Step 2 it follows that there exists $T_{*}<+\infty$ a.s. such that $\Delta_{s}>0$ for all $s \geq T_{*}$. After time $T_{*}$ the system is essentially divided into two half space systems. A virtual right-particle born after time $T_{*}$ (necessarily at site 1 ) will then follow the path of a simple random walk, possibly slowed down by the environment, until it first reaches site 0 , when it will be annihilated; in particular, hitting probabilities for virtual particles are given by a standard gambler's ruin estimate.

Let $K$ be a positive integer. Consider all virtual particles in $[2,+\infty)$ at large times $t>T_{*}$. They can be subdivided into three classes:

- Those which were already present at time $T_{*}$, of which there are finitely many;

- Those which were created after time $T_{*}$ and entered the half-line $[K,+\infty)$ by time $t$. The number of such particles is almost surely asymptotically in $t$ bounded above by $(1+\varepsilon) t / K$, since the environment can possibly slow virtual particles down, but does not affect their trajectories.

- Those which were created after time $T_{*}$, but never exited the box $[1, K]$. From Lemma 1 we get that the number of particles at any given site (except for 0 and 1) behaves sub-linearly - notice that, since the proof of Lemma 1 is purely local, it still applies here to any site that is not directly affected by the additional nucleation at $e_{0}$, which is the case here. Thus, the number of such particles at any $x$ in the box is a.s. less than $t / K^{2}$ for all $t$ large enough. In particular, the total number of such particles is smaller than $t / K$ for all $t$ large enough.

Thus, taking $K$ arbitrarily large we obtain the statement.

Step 4. Observe, that for any $t<+\infty$ there exists $0<R(t)<+\infty$, random, such that $\tilde{h}_{t}(<x, x+1>)=0$ if $|x| \geq R$. Thus, if $\lambda_{0}>3+\lambda$, Step 3 implies that $\tilde{h}_{t}(e)$ grows sub-linearly in time for all $e \neq e_{0}$. Putting this together with Proposition 1 we get that

$$
\lim _{t \rightarrow \infty} \frac{h_{t}^{\prime}(e)}{t}=\lambda, \quad e \neq e_{0},
$$

for such values of $\lambda_{0}$. By the monotonicity of the height dynamics, this immediately extends to all $\lambda_{0}>0$. 
Step 5. Applying the same line of reasoning as in Step 1 we see that $\max \left\{\Delta_{t}, 0\right\}$ grows at most sub-linearly if $\lambda_{0} \leq 1$. Putting together with Step 4, it follows that

$$
\lim _{t \rightarrow \infty} \frac{h_{t}^{\prime}\left(e_{0}\right)}{t}=\lambda, \quad \text { if } \lambda_{0} \leq 1
$$

Step 6. The height process $h_{t}^{\prime}\left(e_{0}\right)$ grows by one with rate at least $\lambda+\lambda_{0}$, and can only decrease if a particle (of the appropriate type) jumps across the edge $e_{0}$ which happens with rate at most 1 . This implies that, if $\lambda_{0}>1$, it is bounded below by a random walk with drift $\lambda+\lambda_{0}-1$. On the other hand, the conclusion of Step 4 implies that for all $e \neq e_{0}, h_{t}^{\prime}(e)$ satisfies a law of large numbers with speed $\lambda<\lambda+\lambda_{0}-1$, thus for all $t$ large enough $n_{t}^{\prime}(0)$ and $n_{t}^{\prime}(1)$ are strictly positive, and so $\Delta_{t}$ as well. Putting these two facts together and using the same reasoning as above, we see that the increase and decrease rates for $h_{t}^{\prime}\left(e_{0}\right)$ become exactly $\lambda+\lambda_{0}$ and 1 , respectively, for large $t$, which implies the stated law for large numbers for $h_{t}^{\prime}\left(e_{0}\right)$, if $\lambda_{0}>1$.

This ends the proof of the main Theorem.

Acknowledgement. The authors thank the anonymous referees for many suggestions and for pointing out an error in the previous version. We also thank prof. M. Myllys for the picture shown as Figure 1. V.B. was partially supported by ANR Project Blan06-3-134462, V.S. and M.E.V. were partially supported by Faperj grant E-26/100.626/2007 APQ1, and CNPq Projeto Universal 48071/2006-1 and individual CNPq grants 300601/2005-0 and 302796/2002-9 respectively.

\section{References}

1. Aldous, D., Diaconis, P.: Hammersley's interacting particle process and longest increasing subsequences. Probab. Theory Related Fields 103(2), 199-213 (1995)

2. Arias-Castro, E., Candes, E., Helgason, H., Zeitouni, O.: Searching for a trail of evidence in a maze. Preprint (2007) arXiv:math.ST/0701668v1

3. Arratia, R.: The motion of a tagged particle in the simple symmetric exclusion process on Z. Ann. Probab. 11(2), 362-373 (1983)

4. Baik, J., Deift, P., Johansson, K.: On the distribution of the length of the longest increasing subsequence of random permutations. J. Amer. Math. Soc. 12(4), 1119-1178 (1999)

5. Baik, J., Rains, E.M.: Symmetrized random permutations. In: Random matrix models and their applications, Math. Sci. Res. Inst. Publ., vol. 40, pp. 1-19. Cambridge Univ. Press, Cambridge (2001)

6. Beffara, V., Sidoravicius, V.: in preparation (2008)

7. Beffara, V., Sidoravicius, V., Spohn, H., Vares, M.: Polymer pinning in random medium as influence percolation. In: F. Den Hollander, E. Verbitsky (eds.) Dynamics and Stochastics, IMS Lecture Notes Monograph Ser, vol. 48, pp. 1-15. Inst. Math. Statist (2006)

8. Chayes, L., Schonmann, R., Swindle, G.: Lifshitz law for the volume of a two dimensional droplet at zero temperature. J. Stat. Phys. 79(4), 821-831 (1995)

9. Janowsky, S.A., Lebowitz, J.L.: Exact results for the asymmetric simple exclusion process with a blockage. J. Statist. Phys. 77(1-2), 35-51 (1994)

10. Kesten, H.: First-passage percolation. In: From classical to modern probability, Progr. Probab., vol. 54, pp. 93-143. Birkhäuser, Basel (2003)

11. Liggett, T.M.: Interacting particle systems, Grundlehren der Mathematischen Wissenschaften, vol. 276. Springer-Verlag, New York (1985)

12. Myllys, M., Maunuksela, J., Merikoski, J., Timonen, J., Horvath, V.K., Ha, M., den Nijs, M.: Effect of columnar defect on the shape of slow-combustion fronts. Preprint (2003). Cond-Mat/0307231 
13. Prähofer, M., Spohn, H.: Scale invariance of the PNG droplet and the Airy process. J. Statist. Phys. 108(5-6), 1071-1106 (2002). Dedicated to David Ruelle and Yasha Sinai on the occasion of their 65 th birthdays

14. Spohn, H.: Interface motion in models with stochastic dynamics. J. Statist. Phys. 71(5-6), 1081-1132 (1993) 